\title{
A test of the survival processing advantage in implicit and explicit memory tests
}

\author{
Dawn M. McBride • Brandon J. Thomas • \\ Corinne Zimmerman
}

Published online: 2 March 2013

(C) Psychonomic Society, Inc. 2013

\begin{abstract}
The present study was designed to investigate the survival processing effect (Nairne, Thompson, \& Pandeirada, Journal of Experimental Psychology: Learning, Memory, and Cognition, 33, 263-273, 2007) in cued implicit and explicit memory tests. The survival effect has been well established in explicit free recall and recognition tests, but has not been evident in implicit memory tests or in cued explicit tests. In Experiment 1 of the present study, we tested implicit and explicit memory for words studied in survival, moving, or pleasantness contexts in stem completion tests. In Experiment 2, we further tested these effects in implicit and explicit category production tests. Across the two experiments, with four separate memory tasks that included a total of 525 subjects, no survival processing advantage was found, replicating the results from implicit tests reported by Tse and Altarriba (Memory \& Cognition, 38, 1110-1121, 2010). Thus, although the survival effect appears to be quite robust in free recall and recognition tests, it has not been replicated in cued implicit and explicit memory tests. The similar results found for the implicit and explicit tests in the present study do not support encoding elaboration explanations of the survival processing effect.
\end{abstract}

Keywords Implicit memory $\cdot$ Evolutionary psychology

In the past several years, numerous studies have reported a memory advantage for items processed for relevance in an evolutionary survival scenario, as compared with the many classic encoding tasks that typically enhance memory (e.g., pleasantness rating, self-referential processing, or imagery).

D. M. McBride $(\bowtie) \cdot$ B. J. Thomas $\cdot$ C. Zimmerman Department of Psychology, Illinois State University, Campus Box 4620,

Normal, IL 61790-4620, USA

e-mail: dmmcbri@ilstu.edu
The motivation for such studies has been to examine an adaptive approach to memory functioning. Nairne, Thompson and Pandeirada (2007) suggested that memory processes developed to aid in remembering fitness-relevant information. They argued that memory processes likely evolved to help us solve basic problems such as hunting and gathering food, finding water and shelter, and evading and protecting ourselves from predators. They hypothesized that processing information for its relevance to survival in an ancestral grasslands scenario should provide a memory advantage as compared with other types of processing, because relevance to survival is consistent with the proposed function of memory.

In four experiments, Nairne et al. (2007) compared survival processing with numerous other encoding tasks, including rating relevance to a moving scenario that did not involve survival, a pleasantness-rating task, and a selfreference rating task. In all of their experiments, a memory advantage was found for the survival processing task as compared to the other encoding tasks in free recall and recognition memory tests. Nairne et al. (2007) concluded from these results that it is possible to test a priori hypotheses regarding an evolutionary perspective for memory functioning.

In the years since Nairne et al.'s (2007) original study testing the survival processing advantage, several studies have further confirmed their results with the same, and some additional, comparison encoding tasks (e.g., a bank robbery scenario, Kang, McDermott, \& Cohen, 2008; professor and elderly stereotype scenarios, Otgaar et al., 2011; itemspecific or relational processing, Burns, Burns, \& Hwang, 2011; and a city survival scenario, Weinstein, Bugg, \& Roediger, 2008). Studies have also generalized the survival processing advantage to picture stimuli (Otgaar, Smeets, \& van Bergen, 2010) and to location memory (Nairne, VanArsdall, Pandeirada, \& Blunt, 2012). Thus, the survival 
processing advantage appears to be robust and generalizes to different types of stimuli and some other types of memory tasks.

Despite the strength of the survival processing effect shown in many studies, possible boundary conditions on the effect have also been reported. For example, Savine, Scullin and Roediger (2011) failed to find a survival processing advantage for face memory. Furthermore, Tse and Altarriba (2010) failed to find a survival processing advantage when testing with implicit memory tests that were matched with explicit tests that showed effects of survival processing.

In the present study, we further explored survival processing in implicit memory tests in order to build on the work of Tse and Altarriba (2010). One reason to examine survival processing effects in implicit memory tests is to look for extensions of these effects in other forms of memory. Implicit tests often rely on more automatic forms of memory than do explicit memory tests. If memory evolved in part to aid in remembering fitness-relevant information, one might hypothesize that automatic forms of memory (such as implicit memory) would show a survival processing advantage similar to that found for explicit memory tests (free recall, recognition), as automatic memory forms seem to be evolutionarily older forms of memory that lower animal species (e.g., invertebrates) are capable of (e.g., Sanders, 1975). In addition, implicit memory tests have been shown to use few or no attentional resources (e.g., Lozito \& Mulligan, 2010). Thus, one might argue that it would be adaptive to have automatic forms of memory attuned to fitness-relevant aspects of the environment. This would afford organisms the opportunity to remember important information without putting a strain on attentional resources that might be focused on other aspects of survival. However, typical tests of implicit memory may be lower in ecological validity, as compared with explicit tests of recognition and free recall, and may not connect well with situations faced by early humans. For example, one can imagine our ancestors freely recalling an episode of encountering the tracks of a predatory animal or recognizing the leaves of a plant with poisonous fruit as aids to their survival, whereas it is more difficult to imagine how survival would be enhanced by the types of processes that increase response rates or speeds of response for studied items on typical implicit tests such as stem completion or categorization tasks. Thus, implicit memory tests may not tap into the types of survival-specific problems that memory processes were developed to solve.

Another reason to examine survival processing effects in implicit tests would be to evaluate suggestions that the survival effect found in past studies is due to a specific mnemonic mechanism such as more semantic elaboration or distinctiveness (see Nairne \& Pandeirada, 2008). Kroneisen and Erdfelder (2011) recently reported results implicating elaboration at encoding as a cause of the survival effect. They found that less elaborate survival scenarios diminished the survival encoding advantage. The use of implicit memory tests provides a further test of the semantic elaboration and distinctiveness descriptions of the effect. Perceptual implicit memory tests (such as stem completion) do not typically show effects of semantic elaboration at encoding (Mulligan, 2006; Roediger, Weldon, Stadler, \& Riegler, 1992; Toth, Reingold, \& Jacoby, 1994). Thus, if survival effects are shown on perceptual implicit tests, they are not likely to be due to greater semantic elaboration at encoding. Conceptual implicit tests (such as category production or category verification), however, do typically show effects of semantic processing at encoding (Hamann, 1990; McBride \& Shoudel, 2003; Weldon \& Coyote, 1996). Thus, if survival effects are due to a greater level of semantic processing for the survival scenario as compared with other encoding tasks, a survival processing advantage should be seen in conceptual implicit tests, resulting in a dissociation across perceptual and conceptual implicit tests. In addition, distinctiveness effects are generally viewed as a phenomenon of explicit retrieval (Smith \& Hunt, 2000). Thus, if distinctiveness is a cause of the survival effect, the effect should be limited to explicit memory tests.

Tse and Altarriba (2010) examined survival effects in both perceptual and conceptual implicit memory tests in two experiments that compared the grasslands survival scenario with the typical moving-scenario and pleasantnessrating tasks used in previous studies. Separate groups of subjects completed implicit and explicit memory tests. In Experiment 1, implicit and explicit versions of a perceptual stem completion test were compared, with subjects in the implicit test being asked to complete stems with the first word that they could think of, and those in the explicit test asked to complete stems with words that they remembered from the rating tasks. In Experiment 2, conceptual memory tests were compared, with subjects in the implicit test being asked to judge the concreteness of presented items, and those in the explicit test asked to complete a recognition test for items they had previously rated.

The traditional survival processing advantage was shown in the recognition test in Experiment 2, but an advantage was not found in either of the implicit tests. The explicit stem completion test in Experiment 1 did not show a memory accuracy advantage for the survival study condition, but reaction times in this test did show a speed advantage for items studied in the survival context. Thus, only a weak survival processing advantage was found in the explicit stem completion tests. Tse and Altarriba (2010) concluded that the survival processing effect may not be present in either perceptual or conceptual implicit tests. The lack of a survival advantage in the explicit stem completion rates may 
also suggest that the effect is limited to only certain types of explicit tests, in terms of memory accuracy. Given the nature of the responses in the stem completion tests (completion of word stems), this may be due to the limited ecological validity of this type of test, as described above.

Due to the absence of survival effects found for most of the tests in the Tse and Altarriba (2010) study, a replication of the lack of effects will be important to produce. In addition, some aspects of their study warrant further tests of the survival effect in implicit memory. First, no survival processing advantage was seen in the memory scores for the explicit stem completion task. Because methodological differences distinguished their study from previous ones, it is unclear why a survival processing effect was not found for explicit stem completion rates. For example, their item lists included both concrete and abstract words, whereas past studies have generally used lists of concrete nouns to facilitate the rating tasks for the survival and moving scenarios. Thus, the type of stimuli used by Tse and Altarriba may have weakened the survival effect, as the relevance for abstract items may have been difficult to determine during the encoding task. Because they did find a survival effect in a recognition test using both concrete and abstract words, it is unlikely that this is the only factor that explains their results, but if survival effects are weaker in implicit than in explicit memory tests, this methodological difference may have contributed to their findings. In addition, the accuracy rates for the concreteness test used in Experiment 2 were low, as compared with what is typically seen in other implicit judgment tasks, such as lexical decision. The low accuracy rates may indicate some confusion on the part of the subjects in judging the concreteness of the items, masking any encoding condition effect that might have been present. Finally, the concreteness test chosen for Experiment 2 involved a judgment task, whereas the stem completion task in Experiment 1 involved a production task. Tse and Altarriba pointed out that this task does not rely on item availability, as production tasks do. If the survival effect relies partly on item availability, then judgment tasks (such as concreteness judgments) may not be as sensitive to the effect, whereas production tasks may be. The authors also pointed out that although recognition is a judgment task, it may rely on item availability to a greater degree than do implicit judgment tasks, accounting for the difference in results for the implicit and explicit tasks in their second experiment. Therefore, they suggested the use of a conceptual production task, as a survival effect might be found for these types of tasks.

The present study provided a further test of the survival processing effect in perceptual and conceptual implicit memory tests, while attempting to closely match the methods and stimuli used in past studies that have shown a survival processing advantage in explicit memory tests (e.g., Nairne et al., 2007). In two experiments, we compared implicit and explicit memory for items rated in a survival processing scenario, a moving scenario, and a pleasantness rating task. In Experiment 1, implicit and explicit versions of a perceptual stem completion test were given to different groups of subjects. The target items were chosen from the items used by Nairne et al. (2007) that had shown a survival effect in both free recall and recognition tests. In Experiment 2 , implicit and explicit versions of a conceptual category production test were given to different groups of subjects. Concrete objects from three categories were included as target items. The subjects completed one of the three encoding tasks, a filler task, and then the memory test that they were assigned. The implicit tests were speeded to discourage explicit retrieval in these tests. A posttest questionnaire identified subjects who had explicitly retrieved studied items in the implicit tests (these subjects were replaced) and those who were aware of the connection between the study items and the test cues. These experiments tested the prediction that a survival processing advantage exists in implicit tests, as it does in the explicit memory tests reported in past studies.

\section{Experiment 1}

\section{Method}

Subjects and design A group of 135 subjects completed one of the three study conditions (survival, moving, or pleasantness) in the implicit test condition ( $n=45$ per study condition). Subjects who reported on the posttest questionnaire that they had explicitly retrieved study items in the implicit test were replaced in the experiment $(n=39)$, and the data from these subjects were not analyzed, as it was unclear to what degree they had employed explicit retrieval. An additional 135 subjects completed one of the three study conditions (survival, moving, or pleasantness) in the explicit test condition ( $n=45$ per study condition). All of the subjects were fluent speakers of English.

For the study, we implemented a between-subjects design with the factors of Rating Scenario (pleasantness, moving, or survival) and Retrieval Task (implicit or explicit). The subjects rated stimuli in one of the three scenarios, completed a Sudoku puzzle as a distractor task, and then completed threeletter stems with instructions dependent on retrieval task condition. Subjects in the explicit test condition were asked to complete stems with studied words, whereas subjects in the implicit test condition were asked to complete stems with the first word that they thought of.

Stimuli and materials Two sets of 30 stimuli were used from Nairne et al.'s (2007) study in the rating task. The 
stimuli were typical members of individual categories. The two sets of stimuli were counterbalanced across subjects in assignments to studied and unstudied item conditions. In the retrieval task, the stems for the implicit conditions consisted of the first three letters of stimuli from both sets, plus ten nonoverlapping filler stems. The stems for the explicit conditions were derived only from the set of stimuli presented in the rating task. All stems were unique, and no stimuli had overlapping three-letter stems. In addition, subjects completed five practice trials in both the rating and retrieval tasks. All stimuli were presented individually in white on a black screen using Macintosh computers. The SuperLab software was used to present the stimuli and collect the responses. Paper materials included booklets with Sudoku puzzles and a two-item postexperimental questionnaire for subjects in the implicit conditions.

Procedure Subjects were tested individually in sessions that lasted approximately $20 \mathrm{~min}$. Upon entering the laboratory, each subject was randomly assigned to one of the three rating scenarios and one of the two retrieval tasks. The instructions for the rating task were identical to those used in the study by Nairne et al. (2007) and were as follows:

Survival: In this task, we would like you to imagine that you are stranded in the grasslands of a foreign land, without any basic survival materials. Over the next few months, you'll need to find steady supplies of food and water and protect yourself from predators. We are going to show you a list of words, and we would like you to rate how relevant each of these words would be for you in this survival situation. Some of the words may be relevant and others may not-it's up to you to decide.

Moving: In this task, we would like you to imagine that you are planning to move to a new home in a foreign land. Over the next few months, you'll need to locate and purchase a new home and transport your belongings. We are going to show you a list of words, and we would like you to rate how relevant each of these words would be for you in accomplishing this task. Some of the words may be relevant and others may not-it's up to you to decide.

Pleasantness: In this task, we are going to show you a list of words, and we would like you to rate the pleasantness of each word. Some of the words may be pleasant and others may not-it's up to you to decide.

The stimuli for the rating task were presented for $5 \mathrm{~s}$ each, and subjects rated each stimulus on a 5-point Likert scale for relevance or pleasantness, depending on the condition. A rating of 1 indicated low relevance or pleasantness, and a rating of 5 indicated high relevance or pleasantness. Subjects were told that each stimulus would only appear for a short duration, so they should respond before each stimulus disappeared. Subjects were not warned about the subsequent retrieval task.

After completing the rating task, all subjects completed 2 min of a distractor task that involved Sudoku puzzles. A beep signified the end of the distractor task, at which point the subjects were given instructions for the retrieval task, which involved completing three-letter stems that appeared individually in the middle of a computer screen. Response boxes were located beneath each stem, and the subjects' answers disappeared upon pressing the Enter key after a response. Stems were presented for $4 \mathrm{~s}$ in the implicit conditions and $10 \mathrm{~s}$ in the explicit conditions. In the implicit condition, subjects were instructed to complete each stem quickly with the first word to come to mind. In the explicit condition, subjects were instructed to fill out the stems with words that corresponded to words that they had encountered in the rating task. Subjects had the option of entering $x x x$ if they could not complete the stem in accordance with the instructions. After completing the retrieval task, the subjects in the implicit conditions completed a questionnaire. This questionnaire asked them whether they had noticed anything about the stems that they had completed in the retrieval task, and whether they intentionally completed stems with words from the rating task. Finally, subjects were debriefed.

\section{Results}

Target items were coded as correct if the subject typed in the completion for the exact target item or a plural form of the target item. Minor misspellings were coded as correct.

Implicit task The mean completion rates for target items in the implicit task are shown in Table 1. A two-way analysis of variance (ANOVA) was conducted on the data from implicit-task subjects with Study Condition (survival, moving, or pleasantness) as the between-subjects factor and Item Type (studied and unstudied) as the withinsubjects factor. No main effect of study condition was

Table 1 Mean target completion rates for Experiment 1 by study condition and item type

\begin{tabular}{lll}
\hline & Studied & Unstudied \\
\hline Implicit Task & & \\
Survival & $.52(.02)$ & $.36(.02)$ \\
Moving & $.50(.02)$ & $.41(.01)$ \\
Pleasantness & $.51(.02)$ & $.36(.02)$ \\
Explicit Task & & \\
Survival & $.69(.02)$ & - \\
Moving & $.66(.02)$ & - \\
Pleasantness & $.69(.02)$ & - \\
\hline
\end{tabular}

Standard errors in parentheses 
evident, $F(2,132)<1.0, p=.46$. Nairne et al. (2007) had reported $\eta_{p}^{2}=.09$ for the survival effect in free recall in their Experiment 1. For this effect size, the power for the sample size used in the present experiment to detect a study effect was estimated to be .91, using the GPower program (Erdfelder, Faul, \& Buchner, 1996). Thus, our sample size appears to have been sufficient for detecting effects of a size similar to those previously reported. A main effect of item type was found, $F(1,132)=142.99, p<.001$, with higher mean completions for studied $(M=.51)$ than for unstudied $(M=.38)$ items, illustrating the typical priming effects seen in implicit memory tests. We also found a significant interaction between study condition and item type, $F(2,132)=$ $4.60, p=.01$. Simple-effects analyses indicated that the study condition means differed for unstudied items, $F(2,132)=5.07, p=.01$, but not for studied items, $F(2,132)<1.0, p=.69$. The interaction appears to have been driven by the higher baseline (unstudied) mean for the moving study condition (see Table 1).

For the 135 subjects not reporting use of "explicit" retrieval strategies on the posttest questionnaire, separate oneway ANOVAs were also conducted for subjects classified as being aware of the connection between the study and test items and subjects classified as being unaware (on the basis of responses on the posttest questionnaire). The mean values for each group are presented in Table 2. No study condition effect was found in either analysis: $F(2,101)=1.16, p=.32$, for aware subjects, and $F(2,28)=2.41, p=.11$, for unaware subjects. A main effect of item type did appear in both analyses $-F(1,101)=89.54, p<.001$, for aware subjects, and $F(1,28)=27.68, p<.001$, for unaware subjectsindicating that priming was present for both groups of subjects. As in the analysis with all subjects, an item type by study condition interaction was found for the aware subjects, $F(2,101)=3.34, p=.04$, but no significant interaction was seen in the unaware subjects, $F(2,28)=1.80, p=.18$. However, only two subjects were classified as being

Table 2 Mean target completion rates in the implicit stem completion test for Experiment 1 by study condition, item type, and awareness

n Studied Unstudied

\begin{tabular}{lccc}
\hline Aware & & & \\
Survival & 30 & $.54(.02)$ & $.39(.02)$ \\
Moving & 31 & $.50(.02)$ & $.43(.02)$ \\
Pleasantness & 43 & $.52(.02)$ & $.37(.02)$ \\
Unaware & & & \\
Survival & 15 & $.48(.04)$ & $.28(.02)$ \\
Moving & 14 & $.51(.04)$ & $.39(.02)$ \\
Pleasantness & 2 & $.42(.11)$ & $.20(.06)$ \\
\hline
\end{tabular}

Standard errors in parentheses unaware in the pleasantness condition, and only 31 subjects were classified as unaware overall, so this analysis should be viewed with caution.

Due to a programming error, study ratings and reaction times (RTs) were only recorded for 66 of the 135 subjects (approximately equal numbers of subjects in each study condition). Study ratings and RTs were compared for these subjects in separate one-way ANOVAs. A study condition effect was found for mean ratings, $F(2,65)=8.18, p=.001$, and for study RTs, $F(2,65)=6.48, p=.003$. Bonferroni post hoc tests indicated that ratings were higher for the survival $(M=3.09)$ and pleasantness $(M=3.14)$ tasks (which did not differ significantly, $p>.99$ ) than for the moving task $(M=2.51), p \mathrm{~s} \leq .004$. RTs were significantly slower for the survival task $(M=2,342 \mathrm{~ms})$ than for the moving $(M=2,050 \mathrm{~ms})$ and pleasantness $(M=1,977 \mathrm{~ms})$ tasks, $p \mathrm{~s} \leq .02$. RTs for the moving and pleasantness tasks did not differ, $p>.99$. However, due to the loss of data for this measure, these results should be interpreted with caution.

Explicit task The mean completion rates for target items in the explicit task are shown in Table 1. A one-way ANOVA revealed that explicit memory in the stem cued recall task did not differ as a function of study task, $F(2,132)=1.38$, $p=.26$. As we stated earlier for the study effect in the implicit memory results, power to detect the effect with our sample size was found to be greater than .90 on the basis of the effect size reported by Nairne et al. (2007). The same programming error as in the implicit task resulted in lost rating data for the explicit subjects, and thus our results should be interpreted with caution. Study ratings and RTs were recorded for only 110 of the 135 subjects (approximately equal numbers of subjects in each study condition). Separate ANOVAs were also conducted on study ratings and the study rating RTs. Study ratings differed according to study task, $F(2,107)=9.90, p<.001$. Post hoc tests indicated that study ratings were higher in the survival $(M=3.10)$ and pleasantness $(M=3.24)$ conditions (which did not differ, $p>.99$ ) than in the moving condition $(M=2.57), p \mathrm{~s} \leq .003$. Study rating RTs were not affected by study condition, $F(2,107)=1.09, p=.34$.

\section{Experiment 2}

In an attempt to generalize the findings of Experiment 1 to a set of conceptual memory tests, in Experiment 2 we employed implicit and explicit category production tasks in a similar design to Experiment 1. If the survival effect were due to greater elaboration during the survival encoding conditions, conceptual tests should be sensitive to the effect. 


\section{Method}

Subjects and design A group of 135 subjects completed each of the three study conditions for the category production implicit test ( $n=45$ per study condition). Subjects who reported on the posttest questionnaire that they had explicitly retrieved study items in the implicit test were replaced in the experiment $(n=86)$, and their data were not analyzed, as it was unclear to what degree explicit retrieval was employed by these subjects. An additional 120 subjects completed each of the three study conditions for the explicit test ( $n=40$ per study condition). All subjects were fluent speakers of English. The design of the study was identical to that of Experiment 1 (study condition and retrieval task factors). None of the subjects from Experiment 1 participated in Experiment 2.

Stimuli and materials Two sets of 30 stimuli were used for the study tasks. Twelve of the items in each set were derived from category norms (van Overschelde, Rawson, \& Dunlosky, 2004): Each set contained four members of the fruit, musical instrument, and four-legged animal categories of medium typicality, based on the norming study (mean generation rate from van Overschelde et al., 2004: $M=.44$ ). The other 18 stimuli in each set were sole members of disparate categories that have been presented in past studies of the survival effect (e.g., Nairne et al., 2007). These stimuli were included so as to reduce the likelihood that subjects would notice the connection between the implicit category production test and the study tasks. However, only the 24 target category items in the sets ( 12 per set) were scored in the tests as target responses. For each subject, one set was assigned as studied items, and the other set was assigned as unstudied items. Set assignments were counterbalanced across subjects. In addition, booklets with one category presented at the top of each page were used for the tests.

Procedure The procedure was identical to that of Experiment 1 for the rating task, distractor task, and postexperimental questionnaire (used only for the implicit-task subjects). For the retrieval task, subjects were given booklets that cued each category. Subjects were cued with one category per page for the three target categories: fruits, musical instruments, and four-legged animals. Subjects in the implicit conditions were instructed to generate category exemplars for each category as quickly as possible with the first words to come to mind. Subjects in these conditions had $30 \mathrm{~s}$ to generate items for each category before being instructed to turn the page. Subjects in the explicit conditions were asked to generate exemplars that belonged to each category that they had encountered in the prior rating task. Subjects in these conditions had $90 \mathrm{~s}$ to generate items for each category before being instructed to turn the page. As in Experiment 1, the faster timing for the implicit task was used to discourage explicit retrieval in this task.

\section{Results}

Target items were coded as correct if the subject wrote the correct studied item or a plural form of the item in the space for the correct category. Minor misspellings were coded as correct responses. Nonstudied categorical intrusions were low for the explicit task $(M=1.55)$ and similar across study conditions. Nontarget categorical intrusions were higher for the implicit task $(M=12.06)$, as expected, but were also similar across conditions.

Implicit task A two-way ANOVA with the Study Condition and Item Type factors was conducted on the target production data. Mean production rates for the implicit test are shown in Table 3 . The study condition effect was not significant, $F(2,132)=1.85, p=.16$. On the basis of the effect size reported by Nairne et al. (2007), the power to detect this effect was the same as was reported for Experiment 1: greater than .90 . In addition, an examination of the means in Table 3 shows that, numerically, the highest mean was in the pleasantness condition. The item type effect was significant, $F(1,132)=97.80, p<.001$, such that studied items $(M=.58)$ resulted in more target responses than did unstudied items $(M=.42)$, indicating priming in the category production task. The interaction between study condition and retrieval task was not significant, $F(2,132)<1.00$, $p=.91$. On the basis of the effect size reported above from Nairne et al. (2007), power to detect the interaction with our sample size was estimated to be greater than .90 .

For the 135 subjects not reporting use of "explicit" retrieval strategies on the posttest questionnaire, separate twoway ANOVAs were also conducted for subjects classified as being aware of the connection between the study and test items and those classified as being unaware (on the basis of

Table 3 Mean target production rates for Experiment 2 by study condition and item type
Studied

Pleasantness

Explicit Task

Survival

Moving

Pleasantness

Standard errors in parentheses

Implicit Task
$.56(.02)$

$.59(.02)$

$.61(.02)$

$.39(.02)$

$.42(.02)$

$.44(.02)$

$.74(.03)$

$.70(.02)$

$.74(.02)$
Unstudied

\begin{tabular}{lll} 
Survival & $.56(.02)$ & $.39(.02)$ \\
Moving & $.59(.02)$ & $.42(.02)$ \\
Pleasantness & $.61(.02)$ & $.44(.02)$ \\
Explicit Task & & \\
Survival & $.74(.03)$ & - \\
Moving & $.70(.02)$ & - \\
Pleasantness & $.74(.02)$ & - \\
\hline
\end{tabular}


responses on the posttest questionnaire). The mean values for each group are presented in Table 4. The study condition effect approached significance for the aware subjects, $F(2,89)=2.74, p=.07$, but not for the unaware subjects, $F(2,40)=1.80, p=.18$. We found main effects of item type in both analyses $-F(1,89)=100.57, p<.001$, for aware subjects, and $F(1,40)=11.85, p=.001$, for unaware subjects - indicating that priming was present for both groups of subjects. As in the analysis with all subjects, the item type by study condition interaction was not significant for either group: $F(2,89)<1.0, p=.54$, for aware subjects, and $F(2,40)<1.0, p=.95$, for unaware subjects. However, only nine subjects were classified as being unaware in the moving condition, and only 43 subjects were classified as being unaware overall, so this analysis should be viewed with caution.

Study ratings and RTs were compared for these subjects in separate one-way ANOVAs. Due to an experimenter error, study rating data were not collected for one moving condition subject. A study condition effect was found for mean ratings, $F(2,131)=67.67, p<.001$, but not for study RTs, $F(2,131)<1.00, p=.56$. Bonferroni post hoc tests indicated that ratings were highest for the pleasantness task $(M=3.43)$ and lowest for the moving task $(M=1.82)$. The survival condition $(M=2.53)$ resulted in a study rating mean between those of the other two conditions, all $p \mathrm{~s}<.001$.

Explicit task Mean production rates for target items in the explicit task are shown in Table 3. A one-way ANOVA was conducted to determine whether study condition affected explicit memory in the category production task, but the effect was not significant, $F(2,117)=1.10, p=.34$. On the basis of the effect size used in the power analyses reported above, the power to detect this effect using our sample size was estimated to be .91. Study ratings and RTs were also compared for study conditions in separate one-way ANOVAs. An effect of study condition was found for the study ratings, $F(2,117)=59.90, p<.001$, with ratings in the

Table 4 Mean target production rates in the implicit category production test for Experiment 2 by study condition, item type, and awareness

\begin{tabular}{lccc}
\hline & $n$ & Studied & Unstudied \\
\hline Aware & & & \\
Survival & 28 & $.57(.03)$ & $.37(.03)$ \\
Moving & 36 & $.60(.03)$ & $.44(.03)$ \\
Pleasantness & 28 & $.64(.03)$ & $.43(.03)$ \\
Unaware & & & \\
Survival & 17 & $.54(.03)$ & $.42(.05)$ \\
Moving & 9 & $.47(.05)$ & $.34(.06)$ \\
Pleasantness & 17 & $.55(.03)$ & $.45(.05)$ \\
\hline
\end{tabular}

Standard errors in parentheses pleasantness task $(M=3.29)$ being highest and moving ratings $(M=1.80)$ lowest overall- the survival ratings, $M=2.62$, fell between the other conditions and were significantly different from the other conditions; all $p \mathrm{~s}<.001$. No effect was seen in study rating RTs, $F(2,117)=1.53$, $p=.221$.

\section{General discussion}

Neither Experiment 1 nor 2 showed support for a survival processing effect in the tasks tested, regardless of test instructions (implicit or explicit). The only significant effect with regard to encoding was the study condition by item interaction in Experiment 1, which was driven by the relatively large number of unstudied items generated in the moving condition. Since the unstudied items were not experienced at all by subjects in the experiment, it is unclear how to interpret this high baseline. Thus, the present study does not support the suggestion that survival processing enhances automatic forms of memory, as tested with the implicit tests used in these experiments. These findings are consistent with those reported by Tse and Altarriba (2010) for stem completion and concreteness judgment implicit tests. Their nonsignificant survival effect for implicit memory tests was supported in the present study with a different set of stimuli, a different sample, and a different form of conceptual implicit test (category production in the present study, concreteness judgments in their study). In addition, the power to detect study condition effects was above .80 in all tasks for medium effect sizes. Despite the lack of survival effects in the present study, both of the implicit tests showed typical priming effects, in that target responses were higher for studied than for unstudied items.

The results found in the present study for implicit tests are not consistent with what would be expected if the survival effect were due to a deeper level of semantic processing than is used for the other encoding strategies it has been compared with. Although perceptual implicit tests, such as stem completion, have typically shown no level-ofprocessing effects in past studies (e.g., Jacoby \& Dallas, 1981; Roediger et al., 1992; Toth et al., 1994), conceptual implicit tests have shown such effects (Hamann, 1990; McBride \& Shoudel, 2003; Weldon \& Coyote, 1996). Thus, a level-of-encoding interpretation of the survival effect would predict a dissociation between perceptual and conceptual implicit tests, such that a conceptual test should show the effect, and perceptual tests should not. Contrary to this prediction, Tse and Altarriba (2010) reported no survival effect in their conceptual implicit test, and none was found in the present study for a different conceptual implicit test. One might also expect more categorical intrusions on the category production tasks in the survival conditions 
if elaboration were higher for the survival scenario, but intrusions were similar across study conditions, with the survival conditions resulting in the numerically fewest intrusions in both tasks.

In the present study, we also failed to replicate the survival effect in explicit tests of stem completion and category production, which have shown level-of-processing effects in past studies. Tse and Altarriba (2010) also reported no survival effect in target responses in their explicit stem completion task, but did report faster test responses for this task for items studied in the survival encoding condition than in the other encoding conditions. Thus, they only found weak support for a survival effect in this explicit test. In fact, Jacoby (1991) has argued that memory tasks should not be thought of as "process pure," and that both automatic and controlled processes likely contribute to performance in explicit and implicit tests. Findings that faster responses in memory tests have typically been associated with more automatic forms of memory than with explicit recollection (e.g., Yonelinas, 2002) further complicate the interpretation of this result. The one test that showed a clear survival effect in Tse and Altarriba's study was a standard recognition task, as has been shown in past studies (e.g., Nairne et al., 2007). Therefore, the lack of survival effects in the explicit tests of the present study is also consistent with Tse and Altarriba's results and is inconsistent with a level-of-processing interpretation of the effect.

The lack of a survival effect on the explicit and implicit category production tasks bears on another recently proposed explanation of the effect. Burns et al. (2011) recently suggested that the survival effect in free recall and recognition tasks is due to both item-specific and relational processing. Their experiments showed that similar results were seen for the survival encoding condition and other encoding conditions that relied on both item-specific and relational processing. Past studies have shown that this combination of processing results in a memory advantage on free recall and recognition tests (e.g., Einstein \& Hunt, 1980; Hunt \& Einstein, 1981). Furthermore, Mulligan (2006) has argued that, whereas implicit category production tasks rely on relational processing, explicit category production tasks rely on both item-specific and relational processing. This idea was recently supported in work by Parker, Dagnall and Munley (2012) with category production tasks. Thus, if Burns et al.'s contention that the survival effect involves both types of processing, one would expect to see a strong survival effect in explicit category production, but not in implicit category production. The results for these tasks in the present study did not support this prediction and are, therefore, at odds with Burns et al.'s suggestion. While null results must be interpreted with caution, the power analyses in the present study indicated sufficient power levels to detect effects of the size reported by Nairne et al. (2007) for free recall using the same stimuli that were used in the present Experiment 1.

More generally, the lack of survival effects in the implicit tests and explicit stem completion tests in the present experiments and in Tse and Altarriba's (2010) study are not consistent with the hypothesis stated in the introduction, that the survival effect might generalize to implicit memory tests. The systems purportedly responsible for implicit memory are more automatic in nature and include the most basic forms of learning in operation in lower vertebrates, and even nonvertebrates (Squire \& Knowlton, 1995). Thus, if survival effects are a byproduct of the evolution of memory, one would expect them to be present in the most basic forms of memory, including implicit memory. Related to this idea, Klein, Cosmides, Tooby and Chance (2002) suggested that "dissociations between memory systems are not ... absolute" (p. 306). They argued that priming is an adaptation in the evolution of memory, but that not all priming tasks may rely on the same systems of memory. Thus, different types of implicit memory tasks than those tested here may rely on the relevant system and show survival effects. The main difference in results between the present study and those reported by Tse and Altarriba involve the explicit category tests. In the present Experiment 2 , a cued category production test was used, whereas Tse and Altarriba included a standard recognition test. Thus, a difference across these explicit tests would involve the type of response required by the subject: either an item production response or an identification (old or new) response. Gabrieli et al. (1999) suggested that these two types of tasks (production vs. identification) can be dissociated on several factors and may be subsumed by different memory systems. More recently, Mulligan and Peterson (2008) suggested that production and identification tasks can be dissociated by certain types of attention manipulations. Thus, this distinction might explain the difference in the explicit memory results across the present study and Tse and Altarriba's.

Related to this idea, Geraci and Rajaram (2004) reported that, whereas an implicit category verification task showed effects of a distinctiveness manipulation, an implicit category production task did not show such an effect. These results suggest that if distinctiveness is a cause of the survival effect, a category production task would not be sensitive to survival processing. However, Tse and Altarriba (2010) included a conceptual concreteness verification task, which should be sensitive to effects due to distinctiveness, and found no survival effect for this task. Thus, their results contradict the distinctiveness explanation as well.

Another possibility was discussed earlier: that the tasks included in the present experiments do not connect well with the types of survival situations that memory evolved 
to handle. The generation of items (explicitly or implicitly) through letter stems or category cues is not easily imagined as being helpful in the evolutionary survival scenarios that it has been proposed that memory developed to aid in. However, free recall and recognition may be more ecologically valid for such scenarios, such that the mechanism by which survival effects occur is more helpful in these tests than in the cue-based tasks used in the present study. It is possible that implicit memory is, in fact, affected by survival processing, but only in tasks that are more suited for the survival situations that evoked the development of these memory processes in early humans. Future studies might address this possibility.

In summary, the present study provided a further test of the survival processing advantage in perceptual and conceptual implicit memory tests. As in findings reported by Tse and Altarriba (2010), no survival effect was seen in either implicit test. Furthermore, the survival effect seen in other explicit tests did not replicate in the explicit forms of cued stem completion or category production tests included in the present study. Taken with the results of Tse and Altarriba, the results in the present study suggest that the survival processing advantage is unique to certain types of memory tests (e.g., free recall and recognition) and cannot yet be consistently explained by typical mechanisms of memorial advantages (e.g., depth of processing or distinctiveness). Thus, the focus on survival as a primary purpose of memory may not be ubiquitous to all forms of memory, but may be focused more on the types of explicit tasks that faced our evolutionary ancestors. More work will be needed to discover the extent to which the effect may be limited to these tests.

Author note The authors thank Michael Nigro and Courtney Schreiner for valuable assistance running subjects and coding data. Brandon Thomas is now at the University of Cincinnati.

\section{References}

Burns, D. J., Burns, S. A., \& Hwang, A. J. (2011). Adaptive memory: Determining the proximate mechanisms responsible for the memorial advantages of survival processing. Journal of Experimental Psychology: Learning, Memory, and Cognition, 37, 206-218. doi:10.1037/a0021325

Einstein, G. O., \& Hunt, R. R. (1980). Levels of processing and organization: Additive effects of individual item and relational processing. Journal of Experimental Psychology: Human Learning and Memory, 6, 588-598.

Erdfelder, E., Faul, F., \& Buchner, A. (1996). GPOWER: A general power analysis program. Behavior Research Methods, Instruments, \& Computers, 28, 1-11. doi:10.3758/BF03203630

Gabrieli, J. D. E., Vaidya, C. J., Stone, M., Francis, W. S., ThompsonSchill, S. L., Fleischman, D. A., ... Wilson, R. S. (1999). Convergent behavioral and neuropsychological evidence for a distinction between identification and production forms of repetition priming. Journal of Experimental Psychology: General, 128, 479-498. doi:10.1037/0096-3445.128.4.479

Geraci, L., \& Rajaram, S. (2004). The distinctiveness effect in the absence of conscious recollection: Evidence from conceptual priming. Journal of Memory and Language, 51, 217-230.

Hamann, S. B. (1990). Level-of-processing effects in conceptually driven implicit tasks. Journal of Experimental Psychology: Learning, Memory, and Cognition, 16, 970-977.

Hunt, R. R., \& Einstein, G. O. (1981). Relational and item-specific information in memory. Journal of Verbal Learning and Verbal Behavior, 19, 497-514.

Jacoby, L. L. (1991). A process dissociation framework: Separating automatic from intentional uses of memory. Journal of Memory and Language, 30, 513-541. doi:10.1016/0749-596X(91)90025-F

Jacoby, L. L., \& Dallas, M. (1981). On the relationship between autobiographical memory and perceptual learning. Journal of Experimental Psychology: General, 110, 306-340. doi:10.1037/ 0096-3445.110.3.306

Kang, S. H. K., McDermott, K. B., \& Cohen, S. M. (2008). The mnemonic advantage of processing fitness-relevant information. Memory \& Cognition, 36, 1151-1156. doi:10.3758/MC.36.6.1151

Klein, S. B., Cosmides, L., Tooby, J., \& Chance, S. (2002). Decisions and the evolution of memory: Multiple systems, multiple functions. Psychological Review, 109, 306-329.

Kroneisen, M., \& Erdfelder, E. (2011). On the plasticity of the survival processing effect. Journal of Experimental Psychology: Learning, Memory, and Cognition, 37, 1553-1562.

Lozito, J. P., \& Mulligan, N. W. (2010). Exploring the role of attention during implicit memory retrieval. Journal of Memory and Language, 63, 387-399.

McBride, D. M., \& Shoudel, H. (2003). Conceptual processing effects on automatic memory. Memory \& Cognition, 31, 393-400.

Mulligan, N. W. (2006). Conceptual implicit memory and the item-specific-relational distinction. In R. R. Hunt \& J. B. Worthen (Eds.), Distinctiveness and memory (pp. 183-210). New York, NY: Oxford University Press. doi:10.1093/acprof:oso/ 9780195169669.003.0009

Mulligan, N. W., \& Peterson, D. (2008). Attention and implicit memory in the category-verification and lexical decision tasks. Journal of Experimental Psychology: Learning, Memory, and Cognition, 34, 662-679.

Nairne, J. S., \& Pandeirada, J. N. S. (2008). Adaptive memory: Is survival processing special? Journal of Memory and Language, 59, 377-385. doi:10.1016/j.jml.2008.06.001

Nairne, J. S., Thompson, S. R., \& Pandeirada, J. N. S. (2007). Adaptive memory: Survival processing enhances retention. Journal of Experimental Psychology: Learning, Memory, and Cognition, 33, 263-273. doi:10.1037/0278-7393.33.2.263

Nairne, J. S., VanArsdall, J. E., Pandeirada, J. N. S., \& Blunt, J. R. (2012). Adaptive memory: Enhanced location memory after survival processing. Journal of Experimental Psychology: Learning, Memory, and Cognition, 38, 495-501. doi:10.1037/ a0025728

Otgaar, H., Smeets, T., Merckelbach, H., Jelicic, M., Verschuere, B., Galliot, A. M., et al. (2011). Adaptive memory: Stereotype activation is not enough. Memory \& Cognition, 39, 1033-1041. doi:10.3758/s13421-011-0091-2

Otgaar, H., Smeets, T., \& van Bergen, S. (2010). Picturing survival memories: Enhanced memory after fitness-relevant processing occurs for verbal and visual stimuli. Memory \& Cognition, 38, 23-28. doi:10.3758/MC.38.1.23

Parker, A., Dagnall, N., \& Munley, G. (2012). Encoding tasks dissociate the effects of divided attention on category-cued recall and category-exemplar generation. Experimental Psychology, $59,124-131$ 
Roediger, H. L., III, Weldon, M. S., Stadler, M. L., \& Riegler, G. L. (1992). Direct comparison of two implicit memory tests: Word fragment and word stem completion. Journal of Experimental Psychology: Learning, Memory, and Cognition, 18, 1251-1269.

Sanders, G. D. (1975). The cephalopods. In W. C. Corning, J. A. Dyal, \& A. O. D. Williams (Eds.), Invertebrates learning (Vol. 3) (pp. 1-101). New York, NY: Plenum Press.

Savine, A. C., Scullin, M. K., \& Roediger, H. L., III. (2011). Survival processing of faces. Memory \& Cognition, 39, 1359-1373. doi:10.3758/s13421-011-0121-0

Smith, R. E., \& Hunt, R. R. (2000). The effects of distinctiveness require reinstatement of organization: The importance of intentional memory instructions. Journal of Memory and Language, 4, 431-446.

Squire, L. R., \& Knowlton, B. J. (1995). Memory, hippocampus, and brain systems. In M. S. Gazzaniga (Ed.), The cognitive neurosciences (pp. 825-837). Cambridge, MA: MIT Press.

Toth, J. P., Reingold, E., \& Jacoby, L. L. (1994). Toward a redefinition of implicit memory: Process dissociations following elaborative processing and self-generation. Journal of Experimental Psychology: Learning, Memory, and Cognition, 20, 290-303.

Tse, C.-S., \& Altarriba, J. (2010). Does survival processing enhance implicit memory? Memory \& Cognition, 38, 1110-1121. doi:10.3758/MC.38.8.1110

Van Overschelde, J. P., Rawson, K. A., \& Dunlosky, J. (2004). Category norms: An updated and expanded version of the Battig and Montague (1969) norms. Journal of Memory and Language, 50, 289-335. doi:10.1016/j.jml.2003.10.003

Weinstein, Y., Bugg, J. M., \& Roediger, H. L., III. (2008). Can the survival recall advantage be explained by basic memory processes? Memory \& Cognition, 36, 913-919. doi:10.3758/MC.36.5.913

Weldon, M. S., \& Coyote, K. C. (1996). Failure to find the picture superiority effect in implicit conceptual memory tests. Journal of Experimental Psychology: Learning, Memory, and Cognition, 22, 670-686.

Yonelinas, A. P. (2002). The nature of recollection and familiarity: A review of 30 years of research. Journal of Memory and Language, 46, 441-517. doi:10.1006/jmla.2002.2864 